\title{
Outage performance analysis of non-orthogonal multiple access systems with RF energy harvesting
}

\author{
Hoang Thien Van ${ }^{1}$, Vo Tien Anh ${ }^{2}$, Danh Hong Le ${ }^{3}$, Ma Quoc Phu ${ }^{4}$, Hoang-Sy Nguyen ${ }^{5}$ \\ ${ }^{1,2}$ The Saigon International University (SIU), Ho Chi Minh City, Vietnam \\ ${ }^{3}$ Van Hien University, Ho Chi Minh City, Vietnam \\ ${ }^{4}$ Binh Duong University, Thu Dau Mot City, Binh Duong Province, Vietnam \\ ${ }^{5}$ Eastem International University, Binh Duong Province, Vietnam
}

\begin{tabular}{l} 
Article Info \\
\hline Article history: \\
Received Aug 16, 2020 \\
Revised Mar 4, 2021 \\
Accepted Mar 15, 2021 \\
\hline Keywords: \\
Delay-limited transmission \\
Energy harvesting \\
Log-normal fading \\
NOMA \\
Outage probability \\
PSR-based protocol
\end{tabular}

\begin{abstract}
Non-orthogonal multiple access (NOMA) has drawn enormous attention from the research community as a promising technology for future wireless communications with increasing demands of capacity and throughput. Especially, in the light of fifth-generation (5G) communication where multiple internet-of-things (IoT) devices are connected, the application of NOMA to indoor wireless networks has become more interesting to study. In view of this, we investigate the NOMA technique in energy harvesting (EH) half-duplex (HD) decode-and-forward (DF) power-splitting relaying (PSR) networks over indoor scenarios which are characterized by log-normal fading channels. The system performance of such networks is evaluated in terms of outage probability (OP) and total throughput for delay-limited transmission mode whose expressions are derived herein. In general, we can see in details how different system parameters affect such networks thanks to the results from Monte Carlo simulations. For illustrating the accuracy of our analytical results, we plot them along with the theoretical ones for comparison.
\end{abstract}

This is an open access article under the CC BY-SA license.

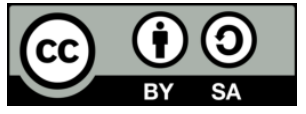

\section{Corresponding Author:}

Danh Hong Le

Van Hien University

Ho Chi Minh City, Vietnam

Email: danhlh@vhu.edu.vn

\section{INTRODUCTION}

NOMA technology is well-known because of its ability to serve multiple users exploiting the same resource block [1], [2]. Additionally, we can find in the existing literature the principal elements of NOMA, which have been well investigated, such as superposition coding, successive interference cancellation (SIC). Thus, it is needless to mention the convenience of deploying NOMA for the massive connectivity need of 5G and IoT applications, [3]-[8]. Besides, simultaneous wireless information and power transfer (SWIPT) systems, as the name suggests, exploit the radio frequency (RF) for EH and transferring data to power finitecapacity batteries in wireless relaying networks, [9]-[14]. Indeed, we can find a wide range of studies relating to EH relaying networks for outdoor scenarios in [15]-[24]. Specifically, in [15]-[17], the two relaying protocols namely power splitting-based (PS) and time switching-based (TS) and their hybrid version in cooperative relaying networks were studied. Additionally, two relay operation modes so-called amplify-andforward (AF) and decode-forward (DF) were investigated in [18]. Especially in [22]-[24], the authors analyzed the system performance of SWIPT networks in the context of NOMA. On the contrary, despite being excellent in modelling the indoor fading variations caused by building walls and moving objects [25]-[27], log-normal fading channels are not studied that intensively comparing to common fading channels 
such as Rayleigh, Nakagami-m. Among the rare study pieces concerning indoor log-normal fading, there were [28-31]. Motivated by the above works, in this paper, we investigate the outage performance of NOMA EH-HD-DF-PSR networks. Differing itself from the existing works, we analyze the networks in indoor scenarios which we model with log-normal fading channels. In section 2, we describe the system model. Section 3 presents the $\mathcal{O P}$ and throughput analytical expressions of the in-studied networks. In section 4 , we discuss the simulation results from the derived expressions. Finally, section 5 concludes our paper.

\section{SYSTEM MODEL}

Depicted in Figure 1(a) is the in-studied system model whereas a source $(S)$ attempts to transmit data to two user devices denoted as $U_{1}$ and $U_{2}$. However, we can see that the $S \rightarrow U_{2}$ communication link cannot be realized because of the in-between obstacle. Thereby, $U_{1}$ is employed to relay the data transmitted from $S$ to $U_{2}$. It should be noted that $U_{1}$ operates in DF mode and is energized by EH the signal that $S$ sends. The $S \rightarrow U_{1}$ and $U_{1} \rightarrow U_{2}$ distances are denoted with $d_{1}$ and $d_{2}$, and assigned with complex channel coefficients of $h_{1}$ and $h_{2}$, respectively. Besides, there are two random variables (RVs) denoted as $\left|h_{1}\right|^{2}$ and $\left|h_{2}\right|^{2}$. The two RVs are independently and identically distributed (i.i.d) over the time block in the log-normal distribution manner, with parameters $\operatorname{LN}\left(\mu_{h_{1}}, \sigma_{h_{1}}^{2}\right)$ and $L N\left(\mu_{h_{2}}, \sigma_{h_{2}}^{2}\right)$, respectively. Additionally, we have $\mu_{h_{1}}$ is the mean value of $10 \log \left(\left|h_{1}\right|^{2}\right)$, and $\sigma_{h_{i}}^{2}$ is the standard deviation of $10 \log \left(\left|h_{i}\right|^{2}\right), i \in\{1,2\}$. With regard to Figure 1(b), we can see the PSR protocol that $U_{1}$ employs in this study for $\mathrm{EH}$ and information transmission (IT) over the time block $T$. Specifically, we have $T$ divided into two $T / 2$ blocks with details below.

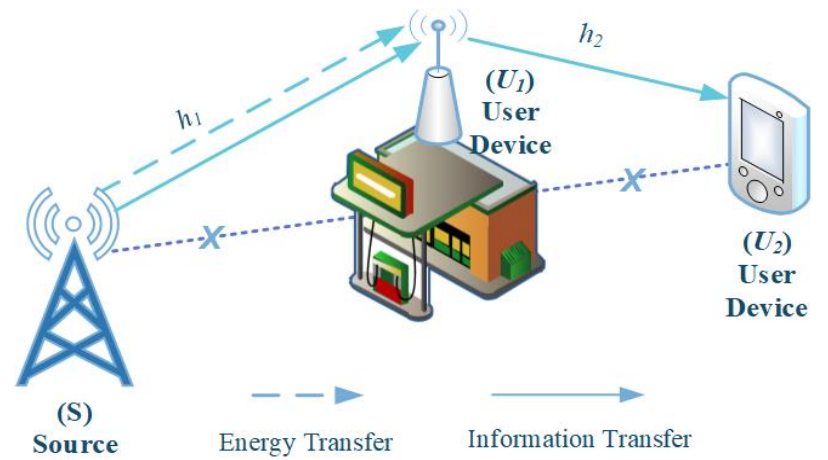

(a)

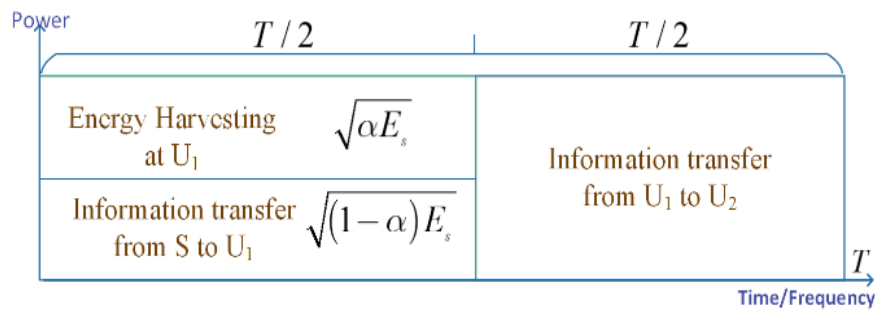

(b)

Figure 1. This figure are; (a) System model, (b) PSR protocol

\subsection{In first time slot}

During the first time slot $T / 2, S$ transmits data with a transmission power of $E_{S}$ to $U_{1}$. The second time slot, remaining $T / 2$, is used for IT from $U_{1}$ to $U_{2}$. As aforementioned, PSR protocol $U_{1}$ separates the $E_{S}$ to two portions for EH and IT purposes. We denote the power splitting (PS) ratio as $\alpha, 0<\alpha<1$. Thereby, at $U_{1}$, the energy amount from $\mathrm{EH}$ process is:

$$
E_{H}=\eta \alpha\left|h_{1}\right|^{2} d_{1}^{-m} E_{S}(T / 2)
$$


where $0<\eta<1$ is the EH efficiency at the energy receiver, determined by the rectifier and EH circuitry that $U_{1}$ deploys. Employing the superposition property of $S^{\prime} s$ transmit signal in NOMA scheme $[1,2]$, the received signal at $U_{1}$ is expressed as shown in (2).

$$
y_{U_{1}}=h_{1}\left(\sqrt{a_{1} E_{S} d_{1}^{-m}} x_{1}+\sqrt{a_{2} E_{S} d_{1}^{-m}} x_{2}\right)+n_{U_{1}},
$$

where we have $a_{1}$ and $a_{2}$ are, respectively, the power allocation coefficients of target signal $x_{1}$ and $x_{2}$ that $S$ attempts to send to $U_{1}$ and $U_{2}$. Besides, we have the additive white Gaussian noise (AWGN) at $U_{1}$, which is $n_{U_{1}}$, with variance $N_{0}$. We assume that $E\left[x_{1}^{2}\right]=E\left[x_{2}^{2}\right]=1$. Since $U_{2}$ is further from $S$ than $U_{1}$, it is allocated with more power. Thus, we have $a_{2}>a_{1}>0$, which satisfies $a_{1}+a_{2}=1$. Additionally, the $U_{1}$ consumes a portion of the energy harvested for its operation while the rest is utilized for DF the received signal to $U_{2}$. Thereby, we can express the transmission power at $U_{1}$ with regard to the harvested energy $E_{H}$ as shown in (3).

$$
E_{U_{1}}=\frac{E_{H}}{(T / 2)}=\frac{\eta \alpha\left|h_{1}\right|^{2} d_{1}^{-m} E_{S}(T / 2)}{(T / 2)}=\eta \alpha\left|h_{1}\right|^{2} d_{1}^{-m} E_{S} .
$$

Considering (2), we have the received signal-to-interference-plus-noise ratio (SINR) at $U_{1}$ for detecting the signal $x_{2}$ of $U_{2}$ formulated in (4).

$$
\gamma_{U_{1}, x_{2}}=\frac{(1-\alpha)\left|h_{1}\right|^{2} d_{1}^{-m} a_{2} \delta}{(1-\alpha)\left|h_{1}\right|^{2} d_{1}^{-m} a_{1} \delta+1}
$$

where $\delta=E_{S} / \sigma^{2}$ is the transmit signal-to-noise ratio (SNR).

The $U_{1}$ receives then decodes the signal $x_{1}$ and $x_{2}$ from $\mathrm{S}$ with the help of SIC [23]. The received SNR that $U_{1}$ exploits to identify its signal, $x_{1}$ is formulated as (5).

$$
\gamma_{U_{1}, x_{1}}=(1-\alpha)\left|h_{1}\right|^{2} d_{1}^{-m} a_{1} \delta
$$

\subsection{In second time slot}

After decoding signal $x_{2}, U_{1}$ forwards the signal to $U_{2}$. Hence, $U_{2}$ receives the signal of:

$$
y_{U_{2}}=\left(\sqrt{E_{U_{1}} d_{2}^{-m}} x_{2}\right) h_{2}+n_{U_{2}},
$$

where $n_{U_{2}}$ is denoted the additive white Gaussian noise (AWGN) at $U_{2}$, with variance $N_{0}$. We substitute (3) into (6) to obtain:

$$
y_{U_{2}}=\sqrt{\eta \alpha d_{1}^{-m} d_{2}^{-m} \delta} h_{1} h_{2} x_{2}+n_{U_{2}} .
$$

Accordingly, the received SNR at $U_{2}$ is expressed as

$$
\gamma_{U_{2}, x_{2}}=\left|h_{1}\right|^{2}\left|h_{2}\right|^{2} d_{1}^{-m} d_{2}^{-m} \eta \alpha \delta \text {. }
$$

\section{PERFORMANCE ANALYSIS}

3.1. Outage performance at $U_{1}$

In NOMA setup, $U_{1}$ is not subject to any outage event $X$ on condition that both the $x_{1}$ and $x_{2}$ that it receives from $S$ are successfully decoded. Hence, with regard to (4) and (5), we can formulate the $\mathcal{O P}$ of $U_{1}$ as shown in (9).

$$
\mathcal{O P} \mathcal{P}_{1}, X=1-\operatorname{Pr}\left(\gamma_{U_{1}, x_{2}}>\gamma_{t h_{2}}, \gamma_{U_{1}, x_{1}}>\gamma_{t h_{1}}\right),
$$


where $\gamma_{t h_{1}}=2^{2 R_{U_{1}}}-1$ and $\gamma_{t h_{2}}=2^{2 R_{U_{2}}}-1$. To detect the $x_{1}$ and $x_{2}$, the target rates $R_{U_{1}}$ and $R_{U_{2}}$ are deployed, respectively. The probability function is $\operatorname{Pr}\left(\right.$.). In general, the $\mathcal{O P}$ of $U_{1}$, deploying $X$ protocol, is expressed in the Theorem 1 as (10),

Theorem 1.

$$
\mathcal{O} \mathcal{P}_{U_{1}, X}=\frac{1}{2}\left(1+\operatorname{erf}\left[\frac{\xi \ln \left(\max \left(\omega_{1}, \omega_{2}\right)\right)-2 \mu_{h_{1}}}{2 \sqrt{2} \sigma_{h_{1}}}\right]\right)
$$

where $\omega_{1}=\frac{\gamma_{t h_{1}}}{a_{1} d_{1}^{-m}(1-\alpha) \delta}$. and $\omega_{2}=\frac{\gamma_{t h_{2}}}{d_{1}^{-m} \delta(1-\alpha)\left(a_{2}-a_{1} \gamma_{t h_{2}}\right)}$ with $a_{2}>a_{1} \gamma_{t h_{2}}$.

Proof:

We can compute the $\mathcal{O P}$ of $U_{1}$ from (11):

$$
\begin{aligned}
\mathcal{O P} P_{U_{1}, X} & =1-\operatorname{Pr}\left(\left|h_{1}\right|^{2} \geq \max \left(\omega_{1}, \omega_{2}\right)\right) \\
& =\operatorname{Pr}\left(\left|h_{1}\right|^{2}<\max \left(\omega_{1}, \omega_{2}\right)\right)=F_{X}\left(\max \left(\omega_{1}, \omega_{2}\right)\right),
\end{aligned}
$$

where $X=\left|h_{1}\right|^{2}$. We symbolized the cumulative distribution function (CDF) of the RV $X$ as $F_{X}\left(\max \left(\omega_{1}, \omega_{2}\right)\right)$. Because $X$ is distributed following log-normal method, the CDF can be expressed as (12).

$$
F_{X}\left(\max \left(\omega_{1}, \omega_{2}\right)\right)=\frac{1}{2}\left(1+\operatorname{erf}\left[\frac{\xi \ln \left(\max \left(\omega_{1}, \omega_{2}\right)\right)-2 \mu_{h_{1}}}{2 \sqrt{2} \sigma_{h_{1}}}\right]\right)
$$

where we have the error function erf[.] as shown in (13).

$$
\operatorname{erf}(x)=\frac{2}{\sqrt{\pi}} \int_{0}^{x} \exp \left(-t^{2}\right) \mathrm{d} t
$$

we substitute (12) into (11) to prove the correctness of the Theorem 1. The proof ends here.

\subsection{Outage performance at $U_{2}$}

Additionally, $U_{2}$ experiences outage event either when $U_{1}$ fails to detect $x_{2}$ to forward to $U_{2}$ or $x_{2}$ is detected but cannot be recovered by $U_{2}$. Thus, with regard to (4) and (8), the $\mathcal{O P}$ of $U_{2}$ is expressed as (14).

$$
\mathcal{O P} \mathcal{U}_{2, x_{2}}=\operatorname{Pr}\left(\gamma_{U_{1}, x_{2}}<\gamma_{t h_{2}}\right)+\operatorname{Pr}\left(\gamma_{U_{2}, x_{2}}<\gamma_{t h_{2}}, \gamma_{U_{1}, x_{2}}>\gamma_{t h_{2}}\right)
$$

Similarly, the $\mathcal{O P}$ of $U_{2}$, deploying signal $x_{2}$ protocol, as shown in (15). Theorem 2 .

$$
\mathcal{O} \mathcal{P}_{U_{2}, x_{2}}=\frac{1}{2}\left(1+\mathcal{A}+\frac{\xi}{\sqrt{2 \pi \sigma_{h_{1}}^{2}}} \int_{\omega_{2}}^{\infty} \frac{1}{x} \exp \left(-\frac{\left(\xi \ln (x)-2 \mu_{h_{1}}\right)^{2}}{8 \sigma_{h_{1}}^{2}}\right)(1+\mathcal{B}) \mathrm{d} x\right)
$$

where $\mathcal{A}=\operatorname{erfc}\left[\frac{\xi \ln \left(\omega_{2}\right)-2 \mu_{h_{2}}}{2 \sqrt{2} \sigma_{h_{2}}}\right], \mathcal{B}=\operatorname{erf}\left[\frac{\xi \ln \left(\frac{\omega_{3}}{x}\right)-2 \mu_{h_{2}}}{2 \sqrt{2} \sigma_{h_{2}}}\right]$, and $\omega_{3}=\frac{\gamma_{t h_{2}}}{\eta \alpha \delta d_{1}^{-m} d_{2}^{-m}}$.

Proof:

From (14), we can rewrite the $\mathcal{O P}_{U_{2}, x_{2}}$ as a sum of two probabilities as shown in (16). 


$$
\mathcal{O P}_{U_{2}, x_{2}}=\underbrace{\operatorname{Pr}\left(\left|h_{1}\right|^{2}<\omega_{2}\right)}_{\mathcal{R}_{1}}+\underbrace{\operatorname{Pr}\left(\left|h_{1}\right|^{2}>\omega_{2},\left|h_{2}\right|^{2}<\frac{\gamma_{t h_{2}}}{\eta \alpha \delta d_{1}^{-m} d_{2}^{-m}\left|h_{1}\right|^{2}}\right)}_{\mathcal{P}_{2}} .
$$

It is possible to calculate the term $\mathcal{P}_{1}$ from

$$
\mathcal{P}=\operatorname{Pr}\left(\left|h_{1}\right|^{2}<\omega_{2}\right)=\frac{1}{2}\left(1+\operatorname{erf}\left[\frac{\xi \ln \left(\omega_{2}\right)-2 \mu_{h_{1}}}{2 \sqrt{2} \sigma_{h_{1}}}\right]\right) .
$$

Additionally, $\mathcal{P}_{2}$ is calculated deploying the probability density function (PDF) and CDF of the aforementioned $X$ and $Y=\left|h_{2}\right|^{2}$ as shown in (18):

$$
\mathcal{P}_{2}=\int_{\omega_{2}}^{\infty} f_{X}(x) F_{Y}\left(\frac{\gamma_{t h}}{\eta \alpha \delta d_{1}^{-m} d_{2}^{-m}} \frac{1}{x}\right) \mathrm{d} x,
$$

where

$$
f_{X}(x)=\frac{\xi}{2 x \sqrt{2 \pi \sigma_{h_{1}}^{2}}} \exp \left[-\frac{\left(\xi \ln (x)-2 \mu_{h_{1}}\right)^{2}}{8 \sigma_{h_{1}}^{2}}\right]
$$

and

$$
F_{Y}\left(\frac{\gamma_{t h_{2}}}{\eta \alpha \delta d_{1}^{-m} d_{2}^{-m}} \frac{1}{x}\right)=\frac{1}{2}\left(1+\operatorname{erf}\left[\frac{\xi \ln \left(\frac{\gamma_{t h_{2}}}{\eta \alpha \delta d_{1}^{-m} d_{2}^{-m}} \frac{1}{x}\right)-2 \mu_{h_{2}}}{2 \sqrt{2} \sigma_{h_{2}}}\right]\right)
$$

Finally, (17) and (18) are substituted into (16) to obtain the $\mathcal{O P}$ of $U_{2}$, which is (15).

\subsection{Total system throughput}

It should be noted that for delay-limited transmission mode, the received signal is decoded by the destination node one block after another. Additionally, we have $\mathrm{S}$ transmit information with a constant rate of $R_{U_{i}}, i \in(1,2)$, which is determined by the $\mathcal{O P}$ over log-normal fading channels. For $X$ protocol in the delaylimited transmission mode [21], we have the total system throughput as shown in (21).

$$
\tau_{X}=\left(1-\mathcal{O P} \mathcal{P}_{U_{1}, X}\right) R_{U_{1}}+\left(1-\mathcal{O} \mathcal{P}_{U_{2}, x_{2}}\right) R_{U_{2}},
$$

where $\mathcal{O P}_{U_{1}, X}$ and $\mathcal{O P}_{U_{2}, x_{2}}$ are consecutively taken from Theorem 1 and 2. Besides, we have the target rates being $R_{U_{1}}$ and $R_{U_{2}}$, which are respectively the target rates for $U_{1}, U_{2}$ to detect $x_{1}$ and $x_{2}$.

\section{RESULTS AND DISCUSSION}

This section presents the Monte Carlo simulation results of the derived expressions to show how the PS factor and the SNR affect the system performance in PSR NOMA scenario over log-normal fading channels. The simulation results are plotted in addition to the analytical results for comparison. For the simulations, we presume that $\eta=1, m=2, d_{1}=d_{2}=2(\mathrm{~m}), \sigma_{h_{1}}=\sigma_{h_{2}}=4(d B), \mu_{h_{1}}=\mu_{h_{2}}=3(d B)$. Moreover, we use the power allocation coefficients of NOMA for $U_{1}$ and $U_{2}$ being $a_{1}=0.2$ and $a_{2}=0.8$, respectively. Last but not least, we set the target rates as $R_{U_{1}}=2(\mathrm{bps} / \mathrm{Hz})$ and $R_{U_{2}}=1(\mathrm{bps} / \mathrm{Hz})$. Figure 2 shows the $\mathcal{O P}$ versus the PS factor, $\alpha$. In general, we can see that the probability that the outage event happens to $U_{1}$ is significantly higher than that of $U_{2}$. 
The $\mathcal{O P}$ raises constantly to the increase of the PS factor for $U_{1}$. However, for $U_{2}$, the $\mathcal{O P}$ first decreases to its minimum value as PS factor approaches $(0.45)$, then rises to its maximum when the PS factor grows further. Figures 3 and 4 consecutively plot the $\mathcal{O P}$ and the throughput versus the SNR. Specifically, in Figure 3, it is obvious that the higher the SNR, the lower the O P. Besides, in Figure 4, we can observe that the throughput-SNR curve of $U_{1}$ is remarkably better than that of $U_{2}$ starting from $\mathrm{SNR}=2.5(\mathrm{~dB})$. Last but not least, we can see the impact of SNR on the $\mathcal{O P}$ with two different data transmission rates in Figure 5. In particular, for the lower $R_{U_{1}}=R_{U_{2}}=R_{U_{0}}$ value, the system performs better indicated by the fact that the $\mathcal{O P}$-SNR curve converges quicker to the zeroth floor, which indicates $100 \%$ success in data transmission. In closing, we can see that the simulation results fit well the analytical ones showing the accuracy of our derived expressions.

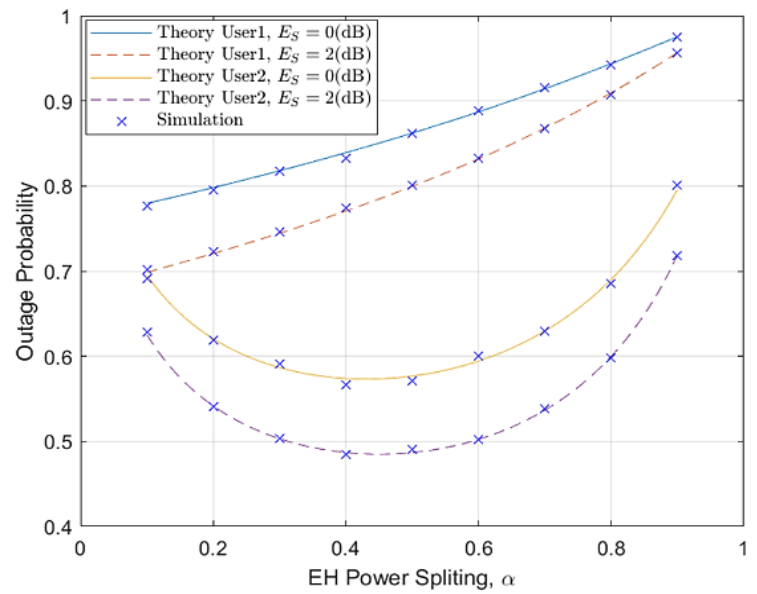

Figure 2. The $\mathcal{O} \mathcal{P}$ performance versus the EH PS factor

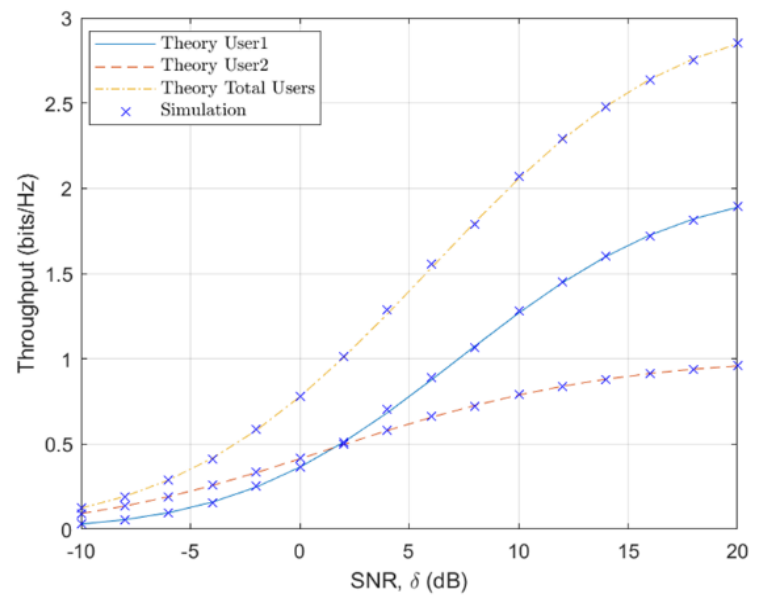

Figure 4. The throughput of two users versus the SNR

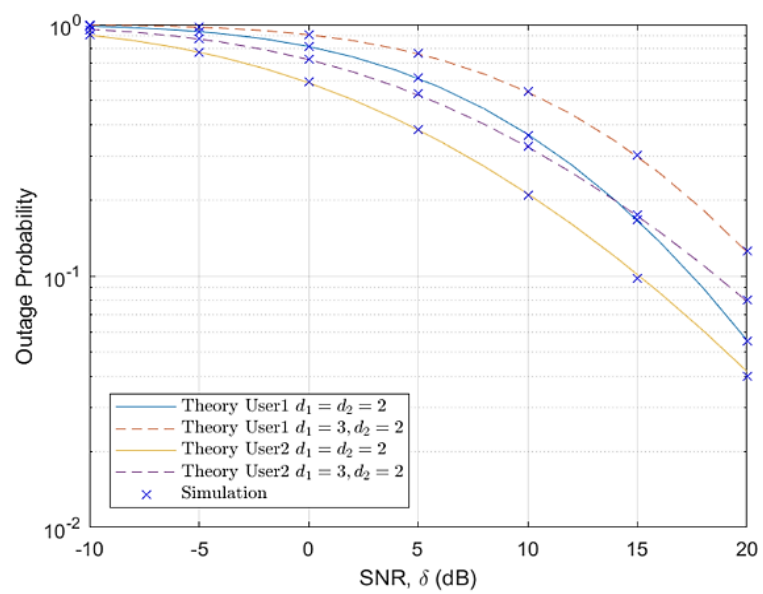

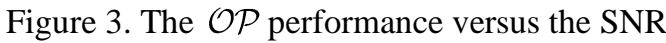

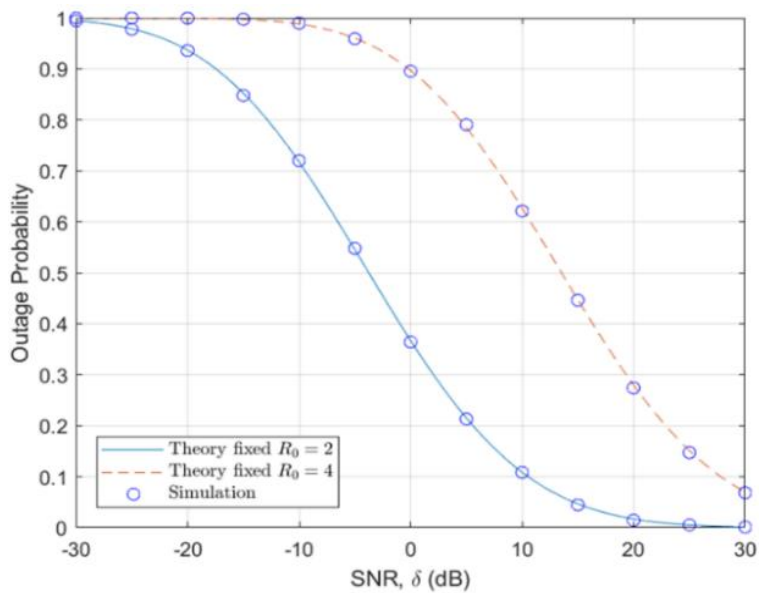

Figure 5. The $\mathcal{O P}$ versus the SNR with two different data transmission rates

\section{CONCLUSION}

In a nutshell, we investigate the $\mathcal{O P}$ and the total throughput for delay-limited transmission mode of NOMA EH-HD-DF-PSR networks over log-normal fading channels. In general, we can conclude that the SNR increase will lead to better throughput, which subsequently makes the $\mathcal{O P}$ smaller. Furthermore, the network performs better with smaller data transmission rate and is more likely to experience outage event as the PS factor becomes higher. 


\section{ACKNOWLEDGEMENTS}

Thanks for the Saigon International University (SIU) funds supporting this project.

\section{REFERENCES}

[1] T. T. H. Ly, H. Sy Nguyen, T. Sang Nguyen, V. V. Huynh, T. Long Nguyen, and M. Voznak, "Outage Probability Analysis in Relaying Cooperative Systems with NOMA Considering Power Splitting," Symmetry, vol. 11, no.1, pp. 1-13, 2019, doi: 10.3390/sym11010072.

[2] Z. Ding, X. Lei, G. K. Karagiannidis, R. Schober, J. Yuan and V. K. Bhargava, "A Survey on Non-Orthogonal Multiple Access for 5G Networks: Research Challenges and Future Trends," in IEEE Journal on Selected Areas in Communications, vol. 35, no. 10, pp. 2181-2195, Oct. 2017, doi: 10.1109/JSAC.2017.2725519.

[3] S. M. R. Islam, N. Avazov, O. A. Dobre and K. Kwak, "Power-Domain Non-Orthogonal Multiple Access (NOMA) in 5G Systems: Potentials and Challenges," in IEEE Communications Surveys \& Tutorials, vol. 19, no. 2, pp. 721-742, Secondquarter 2017, doi: 10.1109/COMST.2016.2621116.

[4] Y. Liu, Z. Qin, M. Elkashlan, Z. Ding, A. Nallanathan and L. Hanzo, "Non-orthogonal Multiple Access for 5G and Beyond," Proceedings of the IEEE, vol. 105, no. 12, pp. 2347-2381, 2017, doi: 10.1109/JPROC.2017.2768666.

[5] Y. Sun, D. W. K. Ng, Z. Ding and R. Schober, "Optimal Joint Power and Subcarrier Allocation for Full-Duplex Multicarrier Non-Orthogonal Multiple Access Systems," in IEEE Transactions on Communications, vol. 65, no. 3, pp. 1077-1091, Mar. 2017, doi: 10.1109/TCOMM.2017.2650992.

[6] Y. Liu, Z. Qin, M. Elkashlan, Y. Gao and L. Hanzo, "Enhancing the Physical Layer Security of Non-Orthogonal Multiple Access in Large-Scale Networks," in IEEE Transactions on Wireless Communications, vol. 16, no. 3, pp. 1656-1672, Mar. 2017, doi: 10.1109/TWC.2017.2650987.

[7] Z. Yang, Z. Ding, P. Fan and N. Al-Dhahir, "A General Power Allocation Scheme to Guarantee Quality of Service in Downlink and Uplink NOMA Systems," in IEEE Transactions on Wireless Communications, vol. 15, no. 11, pp. 7244-7257, Nov. 2016, doi: 10.1109/TWC.2016.2599521.

[8] X. Lu, P. Wang, D. Niyato and Z. Han, "Resource allocation in wireless networks with RF energy harvesting and transfer," in IEEE Network, vol. 29, no. 6, pp. 68-75, Nov.-Dec. 2015, doi: 10.1109/MNET.2015.7340427.

[9] A. Ghasempour, "Internet of Things in Smart Grid: Architecture, Applications, Services, Key Technologies, and Challenges," Inventions journal, vol. 4, no. 1, pp. 1-12, 2019, doi: 10.3390/inventions4010022.

[10] E. Boshkovska, D. W. K. Ng, N. Zlatanov and R. Schober, "Practical Non-Linear Energy Harvesting Model and Resource Allocation for SWIPT Systems," in IEEE Communications Letters, vol. 19, no. 12, pp. 2082-2085, Dec. 2015, doi: 10.1109/LCOMM.2015.2478460.

[11] Y. Zeng and R. Zhang, "Full-Duplex Wireless-Powered Relay With Self-Energy Recycling," in IEEE Wireless Communications Letters, vol. 4, no. 2, pp. 201-204, Apr. 2015, doi: 10.1109/LWC.2015.2396516.

[12] T. D. Ponnimbaduge Perera, D. N. K. Jayakody, S. K. Sharma, S. Chatzinotas and J. Li, "Simultaneous Wireless Information and Power Transfer (SWIPT): Recent Advances and Future Challenges," in IEEE Communications Surveys \& Tutorials, vol. 20, no. 1, pp. 264-302, Firstquarter 2018, doi: 10.1109/COMST.2017.2783901.

[13] A. Ibrahim., M. Iskandar, M. Salem, L. Awalin, A. Jusoh, and T. Sutikno, "Application of inductive coupling for wireless power transfer," International Journal of Power Electronics and Drive Systems (IJPEDS), vol. 11, no. 3, pp. 1109-1116, 2020, doi: 10.11591/ijpeds.v11.i3.pp1109-1116.

[14] M. S. Tamrin and M. R. Ahmad, "Simulation of adaptive power management circuit for hybrid energy harvester and real-time sensing application," International Journal of Power Electronics and Drive Systems (IJPEDS), vol. 11, no. 2, pp. 656-666, 2020, doi: 10.11591/ijpeds.v11.i2.pp658-666.

[15] H.-S. Nguyen, D.-T. Do, and M. Voznak, "Two-way relaying networks in green communications for 5G: Optimal throughput and trade off between relay distance on power splitting-based and time switching-based relaying SWIPT," AEU-International Journal of Electronics and Communications, vol. 70, no. 12, pp. 1637-1644, 2016, doi: 10.1016/j.aeue.2016.10.002.

[16] H.-S. Nguyen., D.-T. Do, T.-S. Nguyen, and M. Voznak, "Exploiting hybrid time switching-based and power splitting-based relaying protocol inwireless powered communication networks with outdated channel state information," Automatika, vol. 58, no. 1, pp. 111-118, 2017, doi: 10.1080/00051144.2017.1372124.

[17] Y. Ye, Y. Li, F. Zhou, N. Al-Dhahir and H. Zhang, "Power Splitting-Based SWIPT With Dual-Hop DF Relaying in the Presence of a Direct Link," in IEEE Systems Journal, vol. 13, no. 2, pp. 1316-1319, Jun. 2019, doi: 10.1109/JSYST.2018.2850944.

[18] H. Nguyen, A. Bui, D. Do and M. Voznak, "Imperfect channel state information of AF and DF energy harvesting cooperative networks," in China Communications, vol. 13, no. 10, pp. 11-19, Oct. 2016, doi: 10.1109/CC.2016.7732008.

[19] Y. Feng, V. C. M. Leung and F. Ji, "Performance Study for SWIPT Cooperative Communication Systems in Shadowed Nakagami Fading Channels," in IEEE Transactions on Wireless Communications, vol. 17, no. 2, pp. 1199-1211, Feb. 2018, doi: 10.1109/TWC.2017.2776933.

[20] H.-S. Nguyen, T.-S. Nguyen, V.-T. Vo, and M. Voznak, "Hybrid full-duplex/half-duplex relay selection scheme with optimal power under individual power constraints and energy harvesting," Computer Communications, vol. 124, pp. 31-44, 2018, doi: 10.1016/j.comcom.2018.04.014.

[21] Y. Ye, Y. Li, L. Shi, R. Q. Hu and H. Zhang, "Improved Hybrid Relaying Protocol for DF Relaying in the Presence of a Direct Link," in IEEE Wireless Communications Letters, vol. 8, no. 1, pp. 173-176, Feb. 2019, doi: 10.1109/LWC.2018.2865476. 
[22] N. T. Do, D. B. Da Costa, T. Q. Duong and B. An, "A BNBF User Selection Scheme for NOMA-Based Cooperative Relaying Systems With SWIPT," in IEEE Communications Letters, vol. 21, no. 3, pp. 664-667, Mar. 2017, doi: 10.1109/LCOMM.2016.2631606.

[23] H.-S. Nguyen.,T. T. Hoc Ly, T.-S. Nguyen, T.-L. Nguyen, and M. Voznak, "Outage performance analysis and SWIPT optimization in energy-harvesting wireless sensor network deploying NOMA," Sensors, vol. 19, no. 3, pp. 1-21, 2019, doi: 10.3390/s19030613.

[24] O. Abbasi, A. Ebrahimi and N. Mokari, "NOMA Inspired Cooperative Relaying System Using an AF Relay," in IEEE Wireless Communications Letters, vol. 8, no. 1, pp. 261-264, Feb. 2019, doi: 10.1109/LWC.2018.2869592.

[25] H. Hashemi, "The indoor radio propagation channel," in Proceedings of the IEEE, vol. 81, no. 7, pp. 943-968, Jul. 1993, doi: 10.1109/5.231342.

[26] A. Laourine, A. Stephenne and S. Affes, "On the capacity of log-normal fading channels," in IEEE Transactions on Communications, vol. 57, no. 6, pp. 1603-1607, Jun. 2009, doi: 10.1109/TCOMM.2009.06.070109.

[27] H. Nouri, F. Touati and M. Uysal, "Diversity-Multiplexing Tradeoff for Log-Normal Fading Channels," in IEEE Transactions on Communications, vol. 64, no. 7, pp. 3119-3129, Jul. 2016, doi: 10.1109/TCOMM.2016.2575019.

[28] K. M. Rabie, A. Salem, E. Alsusa and M. Alouini, "Energy-harvesting in cooperative AF relaying networks over log-normal fading channels," 2016 IEEE International Conference on Communications (ICC), Kuala Lumpur, Malaysia, 2016, pp. 1-7, doi: 10.1109/ICC.2016.7511559.

[29] K. M. Rabie, B. Adebisi and M. Alouini, "Half-Duplex and Full-Duplex AF and DF Relaying With EnergyHarvesting in Log-Normal Fading," in IEEE Transactions on Green Communications and Networking, vol. 1, no. 4, pp. 468-480, Dec. 2017, doi: 10.1109/TGCN.2017.2740258.

[30] R. Katla and A. V. Babu, "Dual-hop full duplex relay networks over composite fading channels: Power and locationoptimization," Physical Communication, vol. 30, pp. 1-14, 2018, doi: 10.1016/j.phycom.2018.07.009.

[31] Y. Liu, R. Xiao, J. Shen, and H. Yang, "Hybrid protocol for wireless energy harvesting network over log-normal fading channel," The Journal of Engineering, vol. 2018, no. 6, pp. 339-341, 2018, doi: 10.1049/joe.2017.0892. 